\title{
Improved GSM-Based Localization by Incorporating Secondary Network Characteristics ${ }^{\star}$
}

\author{
Marek Dvorsky ${ }^{1}$, Libor Michalek $^{1}$, Pavel Moravec $^{2}$, and Roman Sebesta ${ }^{1}$ \\ 1 Department of Telecommunications, FEECS, VŠB - Technical University of Ostrava, \\ 2 Department of Computer Science, FEECS, VŠB - Technical University of Ostrava, \\ 17. listopadu 15, 70833 Ostrava-Poruba, Czech Republic \\ \{marek.dvorsky, libor.michalek, \\ pavel.moravec, roman. sebesta\}@vsb.cz
}

\begin{abstract}
The techniques used in GSM networks for mobile station localization typically use several methods with different level of granularity, based on the base network parameters such as Cell Identification, Timing Advance, the position of Base Transceiver Station, the parameters of Base Transceiver Station antenna etc. This article introduces several others parameters that can be used for network description. This extension can be useful with visualization of localization outputs in cellular network.
\end{abstract}

Keywords: GSM, localization, TA, RXLEV, SRTM.

\section{Introduction}

The most basic localisation methods are based on the Cell Identification (CELL ID) [5, 12]. In Global System for Mobile Communications (GSM) networks [1], Base Transceiver Station (BTS) serves only a limited geographical area. The identity of the BTS serving Mobile Stations (MS) provides the simplest location information. An accuracy improvement of this method is achieved if BTS uses sector antennas.

Additionally, the accuracy of MS localization for GSM BTS, which covers areas over a distance of $550 \mathrm{~m}$, can be improved by limiting area by network parameter Timing Advance (TA) [1]. The TA represents the round trip delay between the MS and the Serving BTS (SBTS). According to the GSM specifications [5], the TA is an integer number between 0 and 63 . The accuracy of this method depends on the size of the covered area, ranging from hundreds of meters to several kilometers [3]. The main benefit of this method is the independence from the type of MS and a low cost of implementation into the real mobile network [7, 13].

The aim of this paper is to describe an extended network parameters, that can be additionally used in the basic location methods in GSM cellular network. It uses measured values of network parameters that contain information about the current network settings and reception conditions in the SBTS and the six Neighbouring BTS (NBTS) [6].

\footnotetext{
* The work was supported by the IT Cluster 2009-2012 No. 5.1 SPK01/029 grant, funded by the European Fund for Regional Development and Ministry of Industry and Trade, and the paper by the BIOM project, reg. No. CZ.1.07/2.3.00/20.0073, funded by Operational Programme Education for Competitiveness, co-financed by ESF and Czech state budget.
} 
Recorded information is then sent by each MS during communication over the GSM cellular network.

\section{Description of Common Parameters}

\subsection{Parameters Provided by Network}

The present localization methods use a set of common parameters provided by the network. In dedicated mode, the MS periodically sends Measurement Report (MR) messages to the network. These messages contain measurements results of parameters defining reception characteristics from the current SBTS and from NBTS cells. The MS can, at most, report the measurements from the 6 strongest BTS. RXLEV of BTS is measured on the Broadcast Control Channel $(\mathrm{BCCH})$ frequencies and with their Base Station Identity Code (BSIC) a Receiving Quality (RXQUAL) parameters are send through Slow Associated Control Channel (SACCH) to the network. For this purpose, so called measurement report is used, see [4].

The TA parameter is provided by network in periodic intervals. TA is decremented or incremented by an air interface one-bit period of about $3.69 \mu \mathrm{s}$. A one bit adjustments corresponds to a radial distance of approximately $550 \mathrm{~m}$ ).

Furthermore, the MS receives information elements about identity of cell, such as CELL ID, Local Area Code (LAC), Mobile Country Code (MCC) and Mobile Network Code (MNC). The purpose of the Cell Identity information element is to identify a cell within a location area. The purpose of the Location Area Identification information element is to provide an unambiguous identification of location areas within the area covered by the GSM network. [4].

\subsection{Parameters Provided by Mobile Telecommunication Operator}

Configuration of each BTS is unique and depends mainly on the location of the station. Each mobile telecommunication operator stores a database of records, which consist of following parameters: Longitude, Latitude, Altitude of BTS; CELL ID; BSIC; BCCH; ID of Base Station Controller (BSC); LAC that identify to which location area the BTS belongs; max. electrical power; azimuth of antenna; tilt of the antenna - the angle from horizontal (from -90 degrees (fold up) to +90 degrees (fold down)) 1 ; the beam width is angular range of the antenna pattern in which at least half of the maximum power is emitted - this angle is then described as beam width or aperture angle (in the GSM network, there are most commonly used antennas with beam width of $60,90,120$ and 360 degrees); the antenna Gain.

\subsection{Measurement Performed by Drive Test}

Drive test is a procedure of recording received levels and timing events whilst driving across a cellular network, e.g. GSM. It is frequently performed during the time when the

\footnotetext{
${ }^{1}$ We distinguish from electrical and mechanical tilt. Electrical tilt is used to reduce signal coverage of a sector area because it improves the close area coverage with increased vertical beam width. BTS mechanical antenna tilt is used as a tool for radio network planners to optimize their networks in sense of coverage and capacity [9].
} 
network is deployed. The reason is that no propagation model can be accurate enough to predict the reception levels in every point of the network. Moreover, the tests are performed also once the deployment of a network is finished. The measurement results can be obtained by performing drive tests with an automatic measurement system, which includes a laptop PC, multi-band GSM test phone and synchronized Global Positioning System (GPS), in order to geo-reference the results.

The trace of drive test should be representative across the network. The trace are chosen according to the concentration of population or according to an importance of place, e.g. motorway. In addition, the measurement results can be also used as an input data for development of a new localization method [8].

\section{Abnormal Propagation Areas}

We can find areas with abnormal wave propagation conditions in the mobile network. Following text describes a base propagation model used at GSM frequency band, typical problematic situations at abnormal areas with connection to a terrain model that is described by Shuttle Radar Topography Mission (SRTM) data.

\subsection{Problematic Places}

When determining mobile node location, the method must take into account a wide range of abnormal situations and conditions, where a general approach which uses combination of several methods is likely to fail or may perform worse than a specific method, suitable only for given situation. Some of these most problematic issues are:

- Signal Reflection - signal reflections cause problems mainly in mountains and heavy urban areas. The problem is reflected in a wrongly reported TA. Obstacles between MS and BTS cause the MS to receive signal reflected from mountains or on other obstacles. As a result, because of the reflected signal the sector is wrongly visualized with radius that has an equal path length as reflected path.

- Side Lobes - one of the antenna parameters is the angle of antenna main lobe $\alpha$ (see chap. 2.2). As a result, the MS is placed aside to main lobe in the direction to so-called side lobe (angle $\alpha+\Delta$ ). In that case the wrongly visualized sector is limited by $\alpha$ range. In this case it should be detected other antenna sectors and their main lobes.

- Repeater - The GSM frequency repeater extends the coverage of a BTS by receiving and re-transmitting signals of the same frequency and at a higher power level. The repeater is a bi-directional amplifier connected to a given BTS.

- Nanocell - the Nanocell BTS improves indoor coverage of a cellular system, e.g. GSM. It is cost effective method that improves coverage and capacity in smaller areas such as conference rooms, sheds, halls etc. In addition, nanocells do not require as accurate network planning due to their low power.

We can exemplify the way abovementioned problems influence the localization in an simple example, shown in Figure 1. In this example, we will use only the combination of RXLEV, TA, BTS antenna position and main lobe direction. 


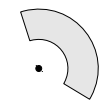

(a)

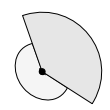

(d)

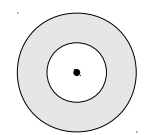

(b)

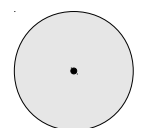

(e)

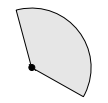

(c)

(f) (a) Main lobe and TA-defined zone available

(b) Only TA-defined zone available

(c) Main lobe and maximum distance

(d) Main lobe and maximum distance with an obstruction causing reflections behind BTS

(e) Only maximum distance

(f) Nanocell and its neighbourhood

Fig. 1. Example of CELL ID, antenna main lobe and TA (or RXLEV/max. distance) combinations

When all required information is available, we can use the localization shown in (a) variant. When the antenna main lobe direction is missing, we have to resort to (b) variant. On the other hand, when we do not have the TA value, or expect it to be invalid, we have to resort to variants (c) or (e) if the angle is not available, with RXLEV-based distance limitation, or limiting the distance to technology limits (e.g. $35 \mathrm{~km}$ ). Should all the parameters be correct, but the reflection is suspected, we can use the pattern $(\mathrm{d})$.

Lastly, should the measured signal come from a nanocell, the distance may be reduced to cover a much smaller area than the TA zone as shown in (f).

\subsection{Digital Elevation Models}

Digital elevation model (DEM) provides the three-dimensional representation of surface obtained from terrain elevation data. They may be represented as a grid, a set of $3 \mathrm{D}$ points or a $3 \mathrm{D}$ mesh. In our approach, we would prefer the digital surface model, since it is easier to measure and includes the buildings.

Operators already have to use commercial digital surface models for coverage calculations, in our case we have decided to use one of the freely-available public resources. We chose the Shuttle Radar Topography Mission (SRTM) data[11], which offers terrain elevation data with the resolution of approximately 90 meters (3") for non-US areas. The SRTMv3 provides sufficient coverage for our main goal, which is the calculation of approximate BTS elevation and detection of mountainous terrain.

\section{Proposed Secondary Attributes}

As mentioned in section 3.1 it is impossible to use a combination of even the basic methods in all situations. Based on available parameters of each individual node in network, we can define a set of parameters which locally describe the whole network. Based on them and expert knowledge, we can define heuristics for correct predicted area visualization.

With the knowledge of network parameters, we can define the first set of secondary attributes:

1. Average distance of $k$-nearest NBTSes - this value can be calculated from network topology, provided by the operator or by obtaining BTS locations form public 
resources. We will not include co-located sectors in this calculation and use this value for determination of BTS density.

2. Intravillan indicator - based on the Average distance of $k$-nearest NBTSes, we can determine, if the BTS is likely to be in intravillan or extravillan environment. The threshold between intravillan and extravillan was set to be $3 \mathrm{~km}$, which corresponds to conducted experiments.

3. Intravillan and Extravillan zones - calculated from the average distances, these indicators may be used to judge the expected location determination precision (especially when using other, independent methods [10]) and for visual improvements in GIS presentation.

4. Borders - state or covered area borders may indicate worse localization performance, since measurement reports may contain unknown combinations of BSICs and BCHs, with both national (not recorded) and foreign BTSes. Also, we may not benefit from techniques based on the inclusion of neighbor lists in this case.

5. Co-located neighbor indication - we should identify, if additional sectors of given site are returned in measurement report. In such case, we should also distinguish between sectors co-located in clockwise and counter-clockwise directions. This information can be used for shifting of the predicted angle (or gradient-based visualization), as well as detection of very close proximity to the BTS (based on RXLEVs and other information in measurement report) or excessive reflections, should colocated sectors in both directions be present.

From the BTS position, antenna azimuth and DEM data, we can determine following two attributes:

6. Antenna elevation - can be used for both BTS visualization (and better operator decision-making) and signal propagation models (especially if the altitude has not been supplied by the operator).

7. Terrain granularity - based on the terrain (and corresponding DEM data) in the direction covered by main antenna lobe, we can determine the terrain granularity. With this information, we can expect and detect the problem. The inspected area can be further fine-tuned by the TA and RXLEV values, similarly to the methods used in methods for modeling of signal propagation [2]. For instance, this can be used to detect mountainous terrain to prevent invalid localizations by using patterns such as Figures $1 \mathrm{~b}$ and $1 \mathrm{~d}$.

Finally, we can include information from actual measurement reports both when doing reference measurements and during the actual localization [10]. Based on these measurements we can define following remaining parameters:

8. Suspicious measurements - BTS data measured during data collection phase together with positions may indicate BTSes where invalid TA values or azimuths were measured. This may be used to prevent invalid visualization and we may use this together with a list of repeater CELL IDs.

9. Broken MS measurement - some values in measurement report may indicate, that it was broken (e.g. the service BTS appears more than once), mostly during the handover. In this case, we have to decide, which pattern from Figure 1 will be used.

10. Nanocell indication - the information may be either provided by operator by special BSC, or by measurements indicating close proximity of BTS but a low RXLEV. 


\section{Conclusion}

In the paper we have developed the set of secondary attributes which will be used together with basic network parameters provided by operators for the selection of correct localization methods and their combination. The resulting attributes could be used as a basis for visualization techniques. In the next step, these attributes will be combined with parameters from other localization method and a resulting model, based both on patterns from Section 3.1 neighboring cell information and vector space model approach [10] will be used to generate the final area.

In future, we could also define additional attributes or use BTS coverage models to better identify possible locations, especially for the UMTS networks, which lack the TA indicator.

\section{References}

1. Ahson, A.S., Ilyas, M.: Location-Based Services Handbook: Applications, Technologies, and Security. CRC Press (2010)

2. Backman, W.: Signal level interpolation for coverage area prediction. In: IEEE 60th Vehicular Technology Conference, VTC 2004-Fall, pp. 67-71 (2004)

3. Eberspächer, J., Vögel, H., Bettstetter, C., Hartmann, C.: GSM-Architecture, Protocols and Service, 3rd edn. John Wiley \& Sons, West Sussex (2002)

4. Digital cellular telecommunications system (phase $2+$ ), mobile radio interface layer 3 specification

5. GSM 04.31 version 8.18 .0 (June 2007)

6. GSM 45.008 version 9.2.0 (April 2010)

7. GSM 08.08 version 8.9.0 (April 2001)

8. Falcone, F., Escauriaza, I.D., Fernandez, A.V., Mau, F.B.: Performance analysis by measurement results in operating 3g network. Wireless Communication Systems, 671-673 (2006)

9. Niemela, J., Lempiainen, J.: Impact of mechanical antenna downtilt on performance of WCDMA cellular network. In: 4th (IEEE 59th) Vehicular Technology Conference, pp. 20912095 (2004)

10. Novosad, T., Martinovic, J., Scherer, P., Snasel, V., Sebesta, R., Klement, P.: Mobile phone positioning in GSM networks based on information retrieval methods and data structures. CCIS (PART 2), vol. 189, pp. 349-363 (2011) cited By (since 1996)

11. Rabus, B., Eineder, M., Roth, A., Bamler, R.: The shuttle radar topography mission a new class of digital elevation models acquired by spaceborne radar. ISPRS Journal of Photogrammetry and Remote Sensing 57(4), 241-262 (2003)

12. Samama, N.: Global Positioning: Technologies and Performance. Wiley-Interscience (2008), Wiley Survival Guides in Engineering and Science

13. Steele, R., Lee, C.C., Gould, P.: GSM, CDMA One and 3G Systems, vol. 1. John Wiley \& Sons, West Sussex (2001) 